\title{
Qilin Pill Exerts Therapeutic Effect on Resection-Induced Premature Ovarian Insufficiency Rats by Inhibiting the MAPK and PI3K-AKT Signaling Pathways
}

\author{
Debao $\mathrm{Li}^{1-3}$ \\ Yuling Jia ${ }^{2,3}$ \\ Yiwen $\mathrm{Hou}^{2,3}$ \\ Dingshi Chen ${ }^{2,3}$ \\ Chiyang Zheng ${ }^{2,3}$ \\ Lifen Chen ${ }^{2,3}$ \\ Li Zhou ${ }^{2,3}$ \\ Zuyue Sun ${ }^{2,3}$ \\ 'School of Pharmacy, Fudan University, \\ Shanghai, 201203, People's Republic of \\ China; ${ }^{2}$ National Health Commission \\ (NHC) Key Laboratory of Reproduction \\ Regulation, Shanghai Institute for \\ Biomedical and Pharmaceutical \\ Technologies, Shanghai, 200032, People's \\ Republic of China; ${ }^{3}$ Reproductive and \\ Developmental Research Institute of \\ Fudan University, Shanghai, 200032, \\ People's Republic of China
}

\begin{abstract}
Background: The Qilin pill (QLP) is a traditional Chinese compound prescription comprising 15 herbs that has demonstrated significant therapeutic effects on premature ovarian insufficiency (POI) in recent years. However, a pharmacological evaluation of QLP on ovarian function remains to be conducted, and the key mechanism of QLP treatment on POI is unclear. Methods: Premature ovarian insufficiency rats were established by bilateral partial ovariectomy. The model rats were administrated with low (QLP-L), medium (QLP-M) and high (QLP-H) doses of QLP for 4 weeks to evaluate the ovarian function in terms of estrous cycle, hormone level, and follicle count. The mechanism of QLP in the treatment of POI was systematically explored by network pharmacology, and expression levels of the MAPK and PI3K-AKT signaling pathways were verified by Western blotting and molecular docking.

Results: The rat model of resection-induced POI was successfully established, and QLP could significantly recover the estrous cycle, decrease serum FSH levels, and decelerate follicle depletion after 4 weeks of administration. The optimal dose of QLP in the experiment was preliminarily determined to be $0.9 \mathrm{~g} / \mathrm{kg}$. Based on the network pharmacology methods, we constructed the compound-target network and protein protein interaction (PPI) network of QLP for the treatment of POI. The experimental verification of the enrichment analysis showed that QLP inhibited the MAPK and PI3K-AKT signaling pathways, and the key compounds and key targets involved were verified by molecular docking.

Conclusion: QLP exerted significant therapeutic effects on resection-induced POI rats, and this was achieved by the inhibition of the MAPK and PI3K-AKT signaling pathways. This study is the first to systematically investigate the effects and mechanism of QLP on POI rats, which will provide valuable guidance in clinic.
\end{abstract}

Keywords: Qilin pill, premature ovarian insufficiency, network pharmacology, MAPK signaling pathway, PI3K-AKT signaling pathway

\section{Introduction}

Premature ovarian insufficiency (POI) is a condition with ovarian dysfunction before the age of 40 in women. ${ }^{1}$ The diagnostic criteria for POI are oligo/amenorrhea for at least 4 months and an elevated FSH level $>25$ IU/L observed on two occasions at an interval of 4 weeks. ${ }^{2}$ Common causes of POI include genetic factors, iatrogenic factors, immune factors, and environmental factors. ${ }^{3}$ Additionally, the etiology of over half of POI cases remains unknown, and this type of POI is called idiopathic POI. ${ }^{4}$ Although
Correspondence: Li Zhou; Zuyue Sun Shanghai Institute for Biomedical and Pharmaceutical Technologies, No. 2140 Xietu Road, Shanghai, 200032, People's Republic of China

Email zhoulijss@163.com;

sunzy64@163.com 
POI exhibits the characteristics of strong heterogeneity, this occurs ultimately due to insufficient primordial follicle reserve, increased follicle exhaustion, and follicular unresponsiveness to gonadotropin stimulation. ${ }^{5}$

Hormone replacement therapy (HRT) is the most common clinical approach used to treat POI. However, the effect of the HRT is limited, and long-term HRT treatment may cause side effects. So, it is imperative to explore and establish effective treatment options for patients with this disease. In recent years, new strategies for POI treatment have attracted people's attention, such as stem cell therapy, ${ }^{6,7}$ platelet-rich plasma (PRP) treatment, ${ }^{8,9}$ and in vitro activation (IVA). ${ }^{10,11}$ Additionally, some ethnic drugs have been emphasized due to good curative effect in clinic. ${ }^{12,13}$ In this study, we focused on the Qilin pill (QLP), a traditional Chinese medicine, and its performance on POI.

Qilin pill (QLP) is used for the clinical treatment of infertility in men and women. It is composed of 15 herbs, including cuscutae semen, lycii fructus, rubi fructus, cynomorii herba, epimedii folium, polygoni multiflori radix preparata, paeoniae radix alba, mori fructus, ecliptae herba, codonopsis radix, astragali radix, dioscoreae rhizoma, citri reticulatae pericarpium viride, salviae miltiorrhizae radix et rhizoma, and curcumae radix. Although several clinical studies have highlighted the exertion of its favorable therapeutic effect on POI patients in recent years, ${ }^{14-16}$ there is a lack of availability of relevant animal experimental studies and systematic exploration of its pharmacological mechanism. Network pharmacology is based on the interaction network of "disease-target-drug" and can be used to systematically investigate the relationship and interactions between multiple drugs and disease targets, which coincides with the multicomponent, multi-pathway, and multi-target theory of traditional Chinese medicine compounds. ${ }^{17}$

In this study, we established a rat model of POI via the surgical resection of bilateral 3/4 ovaries to simulate ovarian surgery-caused POI in clinic and comprehensively explored the effect of QLP on ovarian function. Combined with the results reported by previous studies on the components of QLP, ${ }^{18}$ the compound-target network of QLP in the treatment of POI was constructed using network pharmacology methods to explore the active compounds and potential targets involved and clarify the relationship between drug compounds, targets and the disease. According to the enrichment analysis conducted, the results were further verified. The Western blot analysis showed that QLP inhibited the excessive activation of the MAPK and PI3K-AKT signaling pathways, and the active compounds of QLP were proved to have good binding activity to the key targets enriched in the signaling pathways by molecular docking. This article is a comprehensive and systematic non-clinical study of QLP in treating of POI, which providing an insight into the effect of QLP on ovarian function and the key mechanism involved for the first time. Thus, it provided a meaningful reference to find potential strategies for the treatment of POI.

\section{Materials and Methods}

\section{Animals and Reagents}

A total of 90 specific-pathogen-free female Sprague-Dawley (SD) rats aged 6 months were obtained from Shanghai SuperB\&K Laboratory Animal Corp., Ltd. (Shanghai, China). QiLin pills (lot no. B20180307) were provided by Guangdong Tai'antang Pharmaceutical Co., Ltd. (Guangdong, China). CMC-Na was obtained from Shanghai Aladdin Bio-Chem Technology Co., Ltd. (Shanghai, China). Pentobarbital sodium was obtained from Merck (Darmstadt, Germany). Rat estradiol $\left(\mathrm{E}_{2}\right)$ and follicle-stimulating hormone (FSH) ELISA kits were purchased from Shanghai Yifang Biotechnology Co., Ltd. (Shanghai, China). Primary antibodies against phospho-p44/42 MAPK (p-Erk1/2, \#4370), phospho-SAPK/JNK (\#4668), phospho-p38 MAPK (\#4511), phospho-Akt (Ser473, \#4060), p44/42 MAPK (Erk1/2, \#4695), SAPK/JNK (\#9252), p38 MAPK (\#8690), and Akt (\#4691) were purchased from Cell Signaling Technology (Boston, MA, USA). RIPA lysis buffer, BCA protein assay kit, horseradish peroxidase (HRP)-conjugated rabbit anti-mouse secondary antibody, and anti-GAPDH primary antibody were provided by Shanghai Weiao Biotechnology Co., Ltd (Shanghai, China). All experimental procedures were approved by the Institutional Animal Care and Use Committee of the Shanghai Institute for Biomedical and Pharmaceutical Technologies, and followed the Guide for the Care and Use of Laboratory Animals. ${ }^{19}$

\section{Establishment of the Rat Model with Resection-Induced POI}

Ninety female rats were randomly divided into a sham group $(\mathrm{n}=18)$ and an operating group $(\mathrm{n}=72)$ according to body weight. After intraperitoneal injection of 3\% pentobarbital sodium for anesthesia, a straight incision was performed from the middle of the lower abdomen to gain entry into the abdominal cavity to expose the ovaries. Next, 3/4th portion of the bilateral ovaries was removed from the rats in the operating group, while no surgery was performed on the rats in the sham group (Figure 1). Partial ovariectomy 
was performed by the same experimenter throughout the experiment. After surgery, the changes of rat estrous cycle were observed by daily vaginal smears for one week to evaluate whether the model was successfully established.

\section{Experimental Groups and Administration}

After the successful establishment of the disease model, the operating group animals were randomly and equally divided into four groups ( $\mathrm{n}=18$ per group): the model, lowdose of QLP (QLP-L), medium-dose of QLP (QLP-M) and high-dose of QLP (QLP-H) groups.

The clinical use of QLP is $0.24-0.36 \mathrm{~g} / \mathrm{kg} /$ day, and we took the average dose of $0.30 \mathrm{~g} / \mathrm{kg} /$ day in this study. According to the equivalent dose of rats is approximately six times that of human, the equivalent dose of QLP in rats is $1.8 \mathrm{~g} / \mathrm{kg}^{20}$ Combining the equivalent dose in rats and the results of preexperiments in our laboratory, $0.225,0.9$, and $1.8 \mathrm{~g} / \mathrm{kg}$ were used as the doses in the QLP-L, QLP-M and QLP-H groups, respectively. Due to the poor solubility of QLP, $0.5 \%$ carboxymethylcellulose sodium (CMC-Na) solution was used as the solvent to obtain homogeneous suspension. Since QLP is administered orally in clinic, the rats were administered once a day by gavage. After four weeks of administration, the rats were sacrificed to collect blood samples and ovaries (Figure 1). After dissection, the ovaries of six animals in each group were immediately stored in liquid nitrogen, and the remaining samples from twelve animals were fixed in $10 \%$ formalin solution.

\section{Pharmacological Evaluation}

The animals were observed 1-2 times a day, and their body weight were monitored twice each week. Pharmacological evaluation is mainly based on the estrous cycle, serum hormone level, and follicle count. ${ }^{21,22}$

\section{Estrous Cycle Monitoring}

Daily vaginal smears were performed during administration, and the changes of estrous cycle were evaluated by observing the vaginal epithelial cells. The dropper is slightly inserted into the rat vagina and then the saline flushed into the vagina and back out to collect vaginal epithelial cells. According to the principle of a previous report, the four estrous cycle phases (diestrus, proestrus, estrus, and metestrus) were defined. ${ }^{23}$

\section{Serum $\mathrm{E}_{2}$ and FSH Assay}

Rat blood samples were collected from the abdominal aorta when sacrificed. After centrifugation at $3000 \mathrm{rpm}$ for 15 minutes at $4{ }^{\circ} \mathrm{C}$, the serum was obtained. According to the instructions of rat estradiol $\left(\mathrm{E}_{2}\right)$ and follicle stimulating hormone (FSH) ELISA kit, the serum levels of $\mathrm{E}_{2}$ and $\mathrm{FSH}$ were measured.

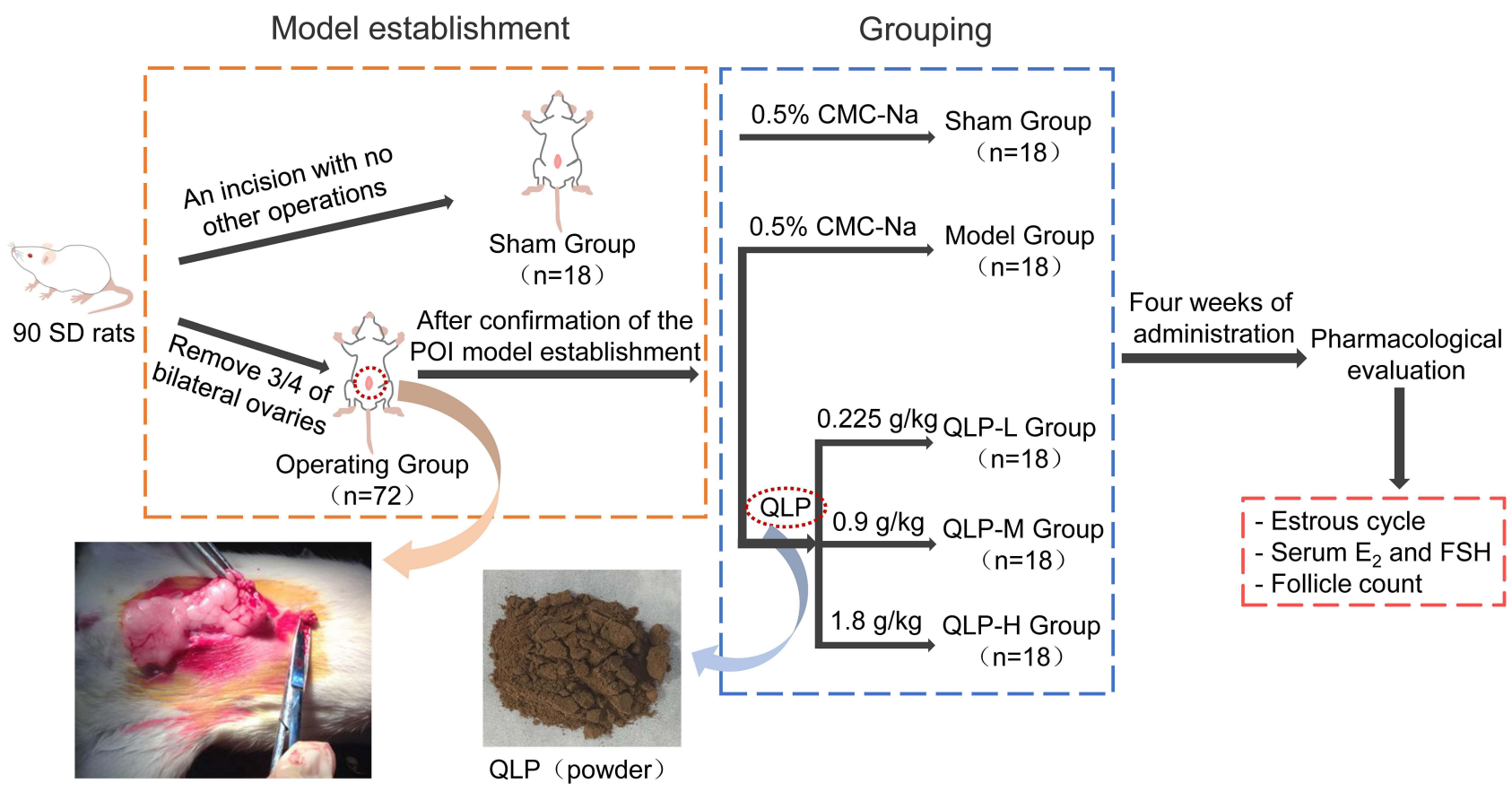

Figure I Graphical summary of the model establishment, grouping, and treatment. 


\section{Follicle Count}

After $48 \mathrm{~h}$ of fixation with $10 \%$ formalin solution, the rat ovarian tissues were embedded in paraffin blocks, cut into 4- $\mu \mathrm{m}$ thick sections, and stained with hematoxylin-eosin (HE). Based on previous studies, the follicles were classified as primordial follicles, primary follicles, secondary follicles, and mature follicles. ${ }^{24,25}$ The number of follicles at different stages in the whole sections was counted using a microscope to visually evaluate the ovarian reserve.

\section{Network Pharmacology of QLP in Treating of POI \\ Chemical Constituents in QLP}

In this study, we obtained the components of QLP by citing the results of the literature on the identification of the chemical constituents of QLP in rat bio-samples after oral administration by ultra-high performance liquid chromatography with quadrupole time-of-flight mass spectrometry (UPLC/ Q-TOF-MS). ${ }^{18}$ The structure of each chemical constituent was illustrated by ChemDraw 19.0 and saved as an SDF format file.

\section{Screening of QLP Targets and POI Targets}

The prediction of QLP targets was based on the results given by the SwissTargetPrediction database (http://www. swisstargetprediction.ch/) and Traditional Chinese Medicine Systems Pharmacology Database and Analysis Platform (TCMSP) (https://tcmspw.com/tcmsp.php). The SDF format file of each chemical constituent structure was loaded into the SwissTargetPrediction database to predict the targets. Meanwhile, the CAS number or English name of each component was used to search for targets in the TCMSP. Combined with the results of the two databases, the targets of QLP were obtained.

The prediction of POI disease targets was based on the DrugBank database (https://go.drugbank.com/), DisGeNET database (https://www.disgenet.org/), and GeneCards database (https://www.genecards.org/).

Additionally, the UniProt database (https://www.uniprot. org/) was used to convert the protein names to Gene Symbol.

\section{The Construction of a Compound-Common Target Network}

The common targets can be obtained through the intersection of QLP targets and POI targets. The relationship between the compounds of QLP and the common targets was imported into the Cytoscape 3.7.2 software to visualize the network.
Protein Protein Interaction Network and Enrichment Analysis

The STRING database (https://string-db.org/) was used to construct the protein protein interaction (PPI) network of common targets. The minimum required interaction score was set to the highest confidence level of 0.900 , and the disconnected nodes in the network were hidden. The data file of the PPI network was imported into Cytoscape 3.7.2 for further analysis. The results of GO enrichment analysis and KEGG pathway enrichment analysis for common targets were also obtained from the analysis module of the STRING database.

\section{Western Blot Analysis}

Based on the findings of enrichment analysis, the most relevant signaling pathways of the therapeutic mechanism were verified by Western blotting.

RIPA lysis buffer containing protease and phosphatase inhibitors was added at a ratio of $100 \mu \mathrm{L}$ of lysis buffer per $20 \mathrm{mg}$ of ovarian tissue. After centrifugation at $12000 \mathrm{rpm}$ for $5 \mathrm{~min}$, the supernatant was collected. The total protein concentration of the supernatant was calculated using the standard curve generated, according to the instructions of the BCA protein assay kit, and the protein concentration of each group was adjusted to the same. The supernatant obtained from each group which was mixed with the same volume of $5 \times$ loading buffer was used as the loading solution. Each loading sample was electrophoretically separated using $10 \%$ SDS polyacrylamide gels and transferred onto polyvinylidene fluoride membranes. The membranes were incubated with primary antibodies at $4^{\circ} \mathrm{C}$ overnight and then incubated with secondary antibodies at room temperature for $2 \mathrm{~h}$ on a decolorizing orbital shaker. Protein bands were detected by performing an electrochemiluminescence method, and the average optical density (IOD) of each band was calculated using Image Pro Plus 6.0.

\section{Molecular Docking}

For the pathways verified by Western blotting, the compound-target-pathway network was constructed using Cytoscape 3.7.2, and the key compound and key targets in the network were selected according to the degree, betweenness centrality, and closeness centrality. Data on the 3D structures of the compounds were downloaded from the PubChem database (https://pubchem.ncbi.nlm.nih.gov/), and data on the target protein structures were downloaded from the PDB database (http://www.rcsb.org/). The Mgltools 1.5.6 software was used for pre-processing of the data on the 
target proteins and ligands, ${ }^{26}$ and the Autodock Vina 1.1.2 software was used to perform the docking process using the processed data on proteins and ligands. ${ }^{27}$

\section{Statistical Analysis}

The results are presented as mean \pm standard error (SE). Statistical analysis was performed using SPSS 25.0. The Student's $t$-test (two groups) or one-way ANOVA (multiple groups) was used for data analysis when data showed a normal distribution and the homogeneity of variance. Otherwise, the Mann-Whitney $U$-test (two groups) or Kruskal-Wallis test (multiple groups) was adopted. The LSD method was used as a post-hoc test after one-way ANOVA, and the Bonferroni method was used after performing the Kruskal-Wallis test. Statistical significance was set at $\mathrm{P}<0.05$.

\section{Results}

\section{Bilateral Partial Ovariectomy Causes POI}

One week after surgery, the diestrus of the operating group was significantly prolonged (Figure $2 \mathrm{~A} ; \mathrm{P}<0.01$ ). More importantly, the number of estrus cycles was significantly decreased (Figure 2B; $\mathrm{P}<0.01$ ). These results indicated that the estrus cycle of the operating group was disordered by bilateral partial ovariectomy, and the POI rat model was successfully established.

\section{Therapeutic Effects of QLP on Resection-Induced POI Rats General Condition and Body Weight}

During the period of administration, the animals in each group demonstrated normal physiological activities and no

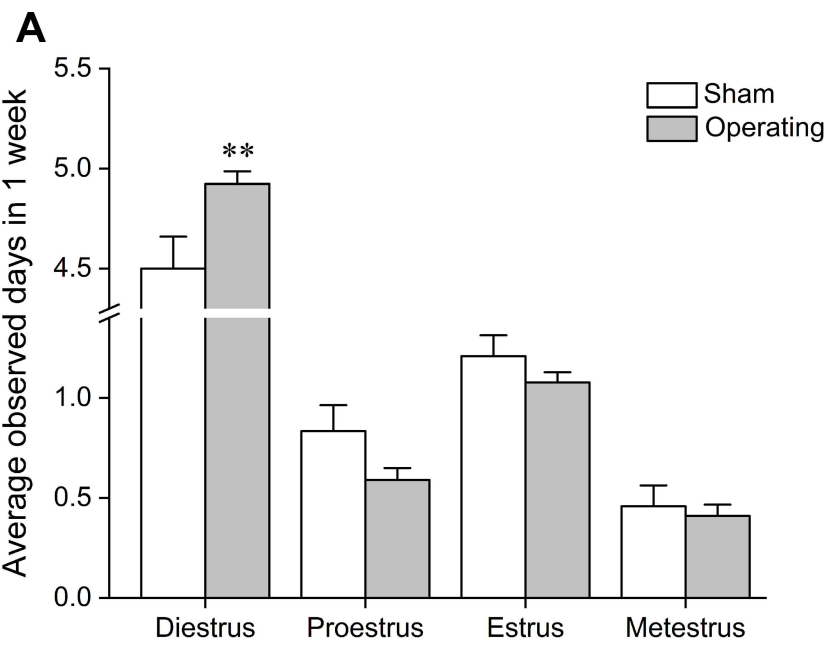

evident changes occurred in their general condition. As shown in Figure 3A, at each point in time, there was no significant difference in body weight $(\mathrm{P}>0.05)$, indicating that QLP exerted no significant effects on the general condition and body weight of rats.

\section{Estrous Cycle}

During 4 weeks of administration, the number of estrous cycles in the QLP-M and QLP-H groups increased obviously compared with the model group, and the QLP$M$ group showed the achievement of statistical significance (Figure $3 \mathrm{C} ; \mathrm{P}<0.05$ ). The number of days in each phase of the estrous cycle in the QLP-M and QLP-H groups was also closer to that in the sham group (Figure 3B), indicating that QLP exerted a positive regulatory effect on the recovery of the estrous cycle in rats.

\section{Serum FSH and $E_{2}$ Levels}

As shown in Figure 3D, the serum FSH levels of the QLP$\mathrm{M}$ and QLP-H groups were lower than those of the sham and model groups. Compared with the sham group, the serum FSH level in the QLP-M group decreased significantly $(\mathrm{P}<0.05)$. Significant differences were observed in FSH levels in the QLP-H group compared with the sham and model groups $(\mathrm{P}<0.05)$.

ELISA showed that the serum $\mathrm{E}_{2}$ level in each group decreased after bilateral partial ovariectomy, and the serum $\mathrm{E}_{2}$ levels in the QLP-M group recovered to a certain extent, but the difference was not significant (Figure 3E).

\section{Follicle Count}

As shown in Figure $3 \mathrm{G}$ and $\mathrm{H}$, compared with the sham group, the number of primordial follicles and total follicles

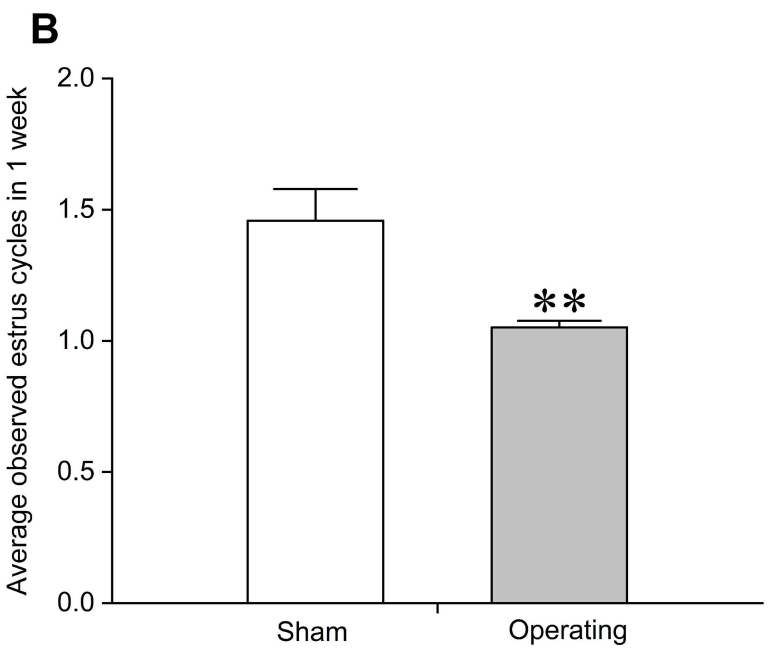

Figure 2 Effects of a bilateral partial ovariectomy on the estrous cycle in female rats. (A) Average observed days of four estrous cycle phases in one week after surgery. (B) Average observed estrus cycles in one week after surgery. $* * \mathrm{P}<0.01$, compared with sham group. $\mathrm{N}=18$ in the sham group, $\mathrm{N}=72$ in the model group. 
A

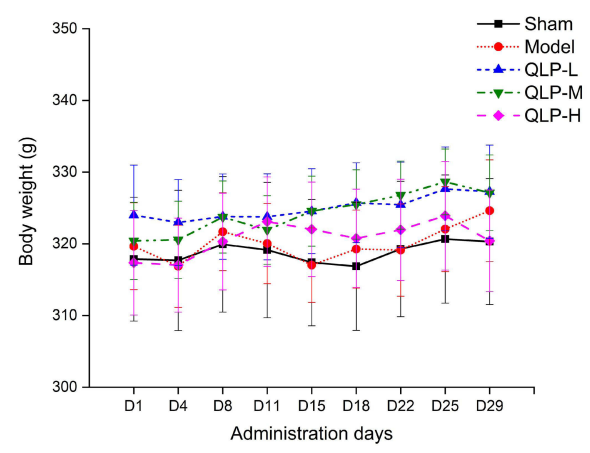

D

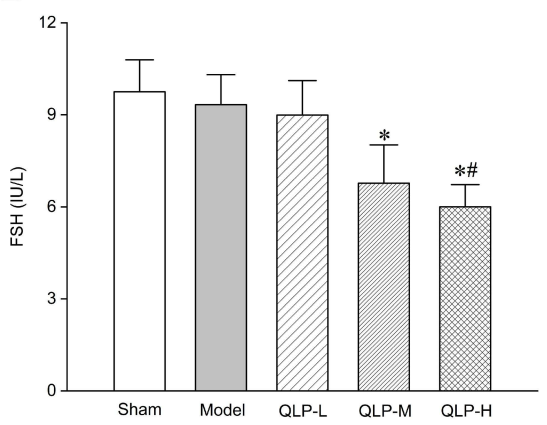

B

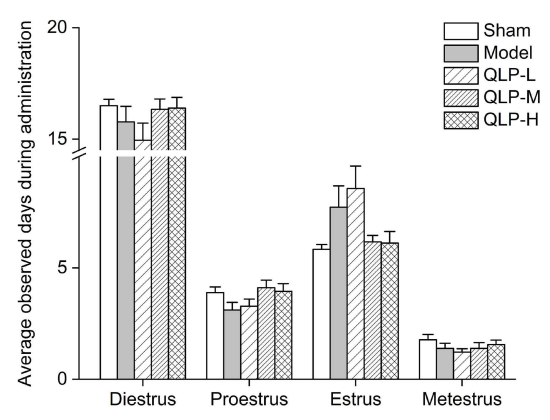

C

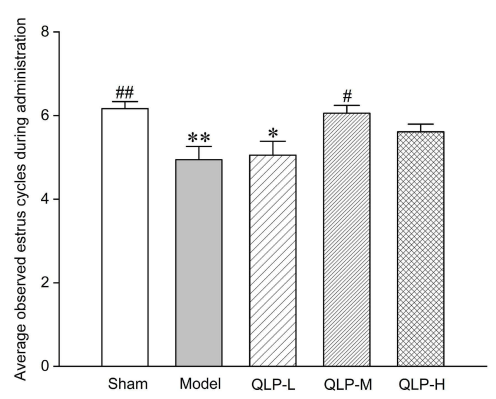

E

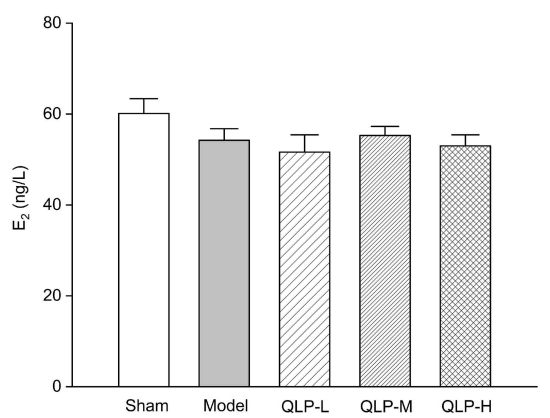

F

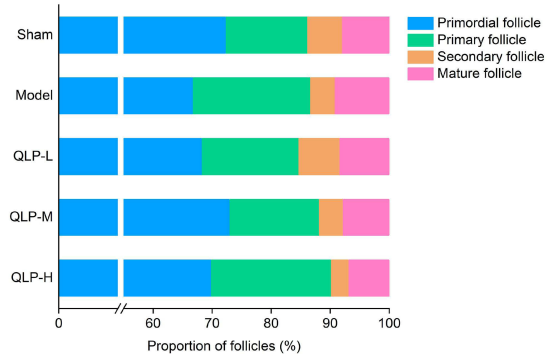

G

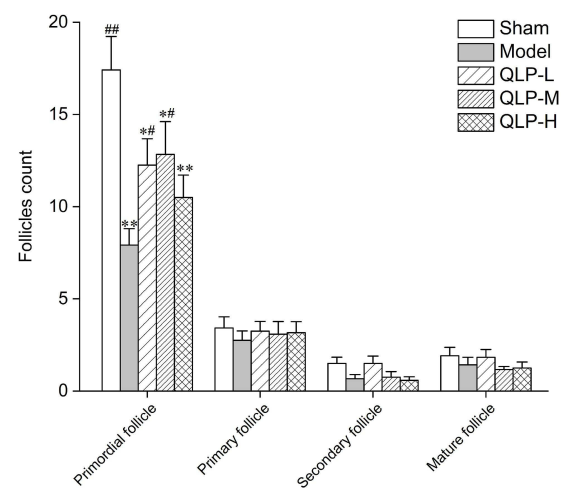

H

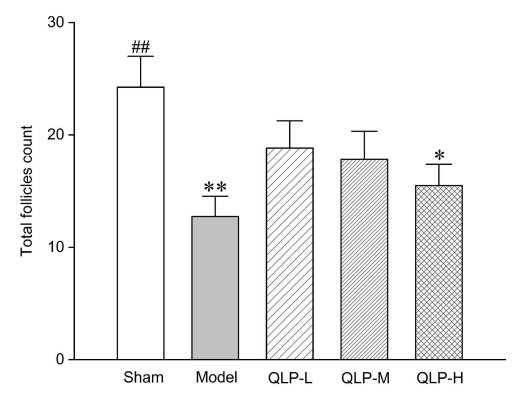

Figure 3 Effects of QLP exerted on resection-induced POI rats after 4 weeks of administration. In each group: (A) body weight changes of rats during 4 weeks of administration; (B) average observed days of four estrous cycle phases during administration; (C) average observed estrus cycles during administration; serum FSH (D) and $\mathrm{E}_{2}$ (E) levels; percentage $(\mathbf{F})$ and number $(\mathbf{G})$ of follicles at different stages; total follicles $(\mathbf{H})$. ${ }^{*} \mathrm{P}<0.05$, ${ }^{* *} \mathrm{P}<0.0 \mathrm{l}$, compared with the sham group; ${ }^{\#} \mathrm{P}<0.05$, ${ }^{\#} \mathrm{P}<0.0 \mathrm{I}$, compared with the model group, $\mathrm{N}=18$ in each group in body weight measurement, estrous cycle monitoring, and serum hormone assay; $\mathrm{N}=12$ in each group in follicle count.

in the model group was significantly decreased $(\mathrm{P}<0.01)$. After 4 weeks of administration of QLP, the primordial follicles and total follicles were increased compared with the model group, reaching statistically significant levels in the QLP-L and QLP-M groups $(\mathrm{P}<0.05)$. The follicles in the sham group were more abundant, reflecting the integrity of the ovarian reserve in the group. Figure $3 \mathrm{~F}$ shows the percentages of follicles at different stages. Compared with the model group, the proportion of primordial follicles increased after treatment, especially in the QLP-M group.

\section{Network Pharmacology Analysis}

\section{Compound-Common Target Network Analysis}

Based on the identified chemical constituents of QLP in rat bio-samples in the existing literature, ${ }^{18}$ a total of 66 compounds were obtained (Supplementary Table 1), excluding isomers and compounds whose structures could not be verified. A total of 474 targets were considered as QLP targets according to the SwissTargetPrediction database and the TCMSP (Supplementary Table 2). 
Meanwhile, data on 679 POI targets were collected from the DrugBank, DisGeNET, and GeneCards databases (Supplementary Table 3). A total of 100 common targets were obtained as therapeutic targets (Supplementary Table 4). The compound-common target network, which consists of 165 nodes and 671 edges, was constructed using Cytoscape 3.7.2 (Figure 4).

\section{PPI Network Analysis}

The PPI network consisted of 88 nodes and 442 edges after the disconnected nodes were hidden. As shown in Figure 5A, the closely related nodes in the PPI network are STAT3, SRC, MAPK3, MAPK1, and PIK3R1.

\section{GO Enrichment Analysis and KEGG Pathway Enrichment Analysis}

GO enrichment analysis aided the identification of 1671 biological process (BP) terms, 112 cellular component (CC) terms, and 183 molecular function (MF) terms. The BP terms mainly included the response to organic substances, cellular responses to chemical stimuli, responses to drugs, and regulation of cell population proliferation. The most enriched CC items mainly included membranebounded organelles, cytoplasmic parts, and endomembrane systems. The most relevant MF terms mainly included protein binding, signaling receptor binding, and phosphotransferase activity. Based on the p-value, the top $10 \mathrm{BP}, \mathrm{CC}$, and MF terms are shown in Figure 5B.
A total of 172 signaling pathways were obtained by KEGG enrichment analysis. The most enriched pathways mainly included pathways in cancer, proteoglycans in cancer, MAPK signaling pathway, PI3K-AKT signaling pathway, Kaposi's sarcoma-associated herpesvirus infection, hepatitis B, and endocrine resistance. The top 20 pathways with the most significant $\mathrm{P}$-values are shown in the enrichment bubble diagram (Figure 5C).

To further investigate the common targets involved in the top 20 pathways, the target-pathway network was constructed using Cytoscape 3.7.2 (Figure 5D). The network clearly shows that the top 20 pathways involved a total of 66 common targets, most of which were enriched in different pathways.

\section{Validation of the MAPK and PI3K-AKT Signaling Pathways}

Based on the results of enrichment analysis and findings reported by previous studies, ${ }^{28}$ it was inferred that QLP most likely exerted therapeutic effects via the MAPK and PI3K-AKT signaling pathways. As shown in Figure 6A and $\mathrm{B}$, the common targets enriched in the MAPK and PI3K-AKT signaling pathways are indicated in red in the Kyoto Encyclopedia of Genes and Genomes (KEGG) database (https://www.kegg.jp/) using KEGG Mapper analysis tools.

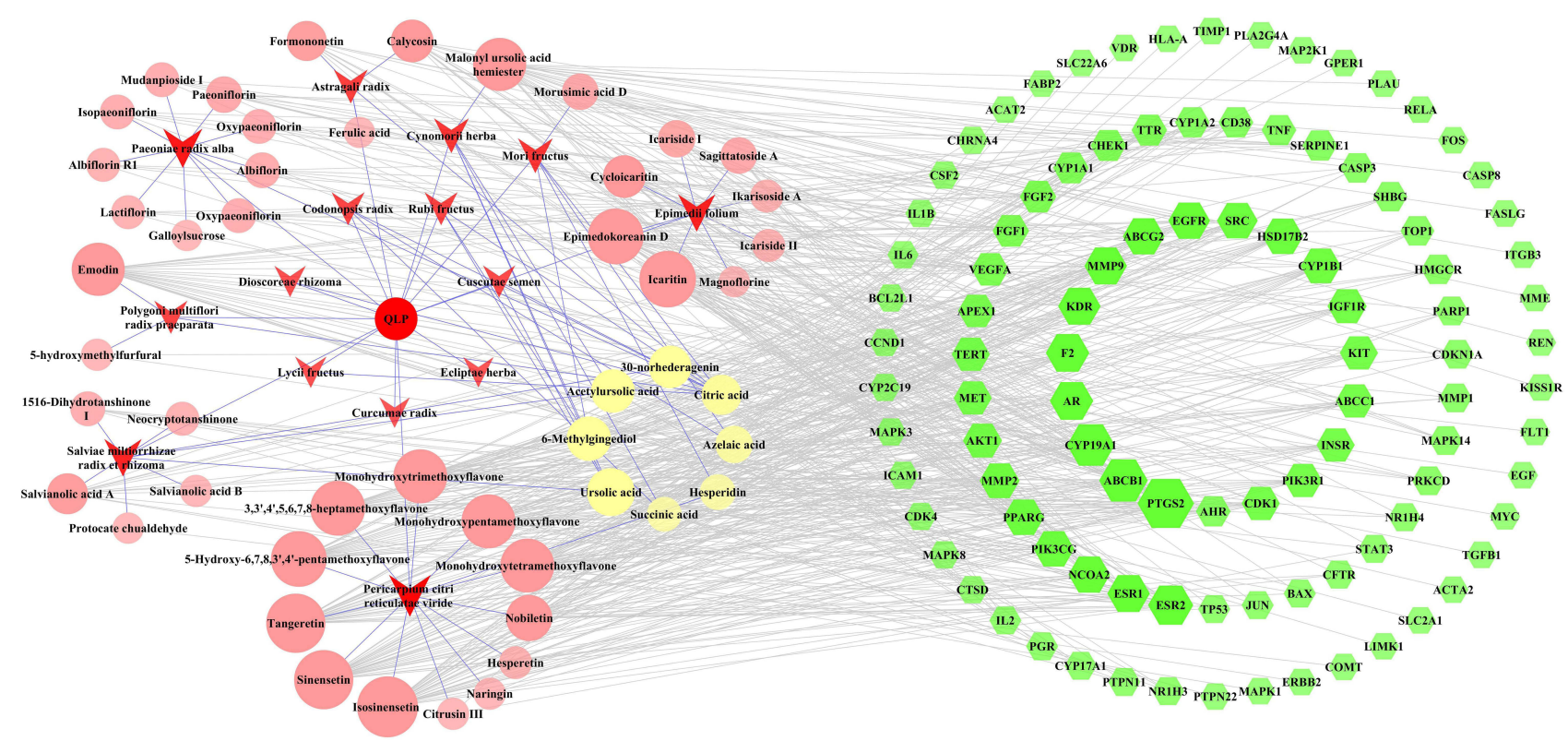

Figure 4 Compound-common target network. The green-colored nodes in the network represent the common targets, the red arrow-shaped nodes indicate the herb medicines of QLP, and the light red- and light yellow-colored nodes represent the compounds of QLP. The light yellow-colored nodes refer specifically to compounds shared by more than one herb. The size of the nodes in the network are determined by the degree value. The greater the degree value, the larger the nodes. 
A

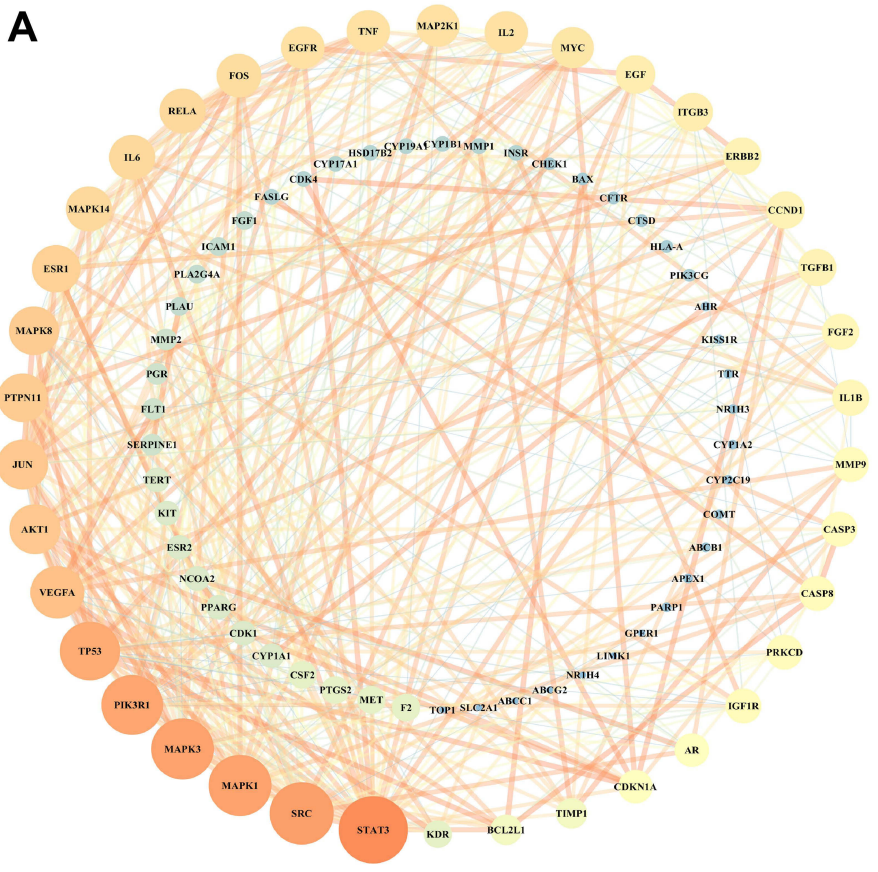

C

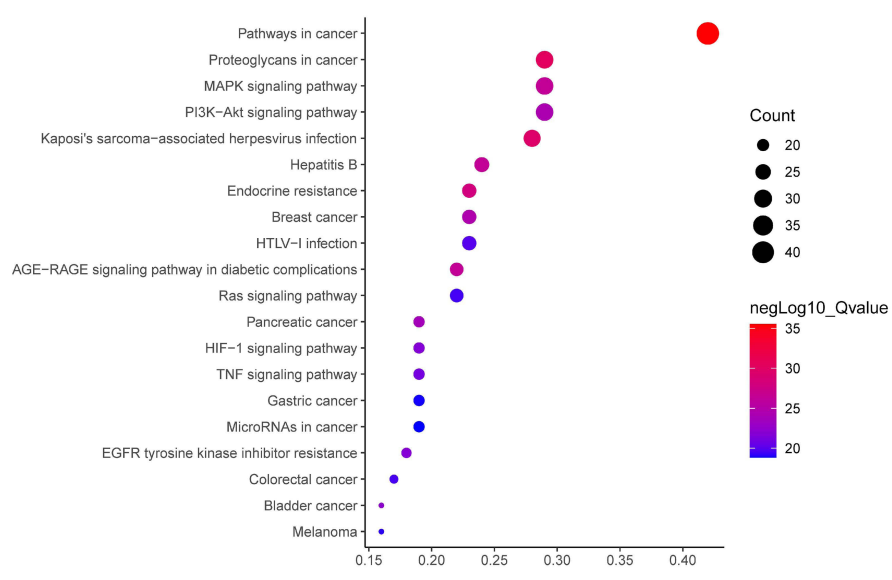

B

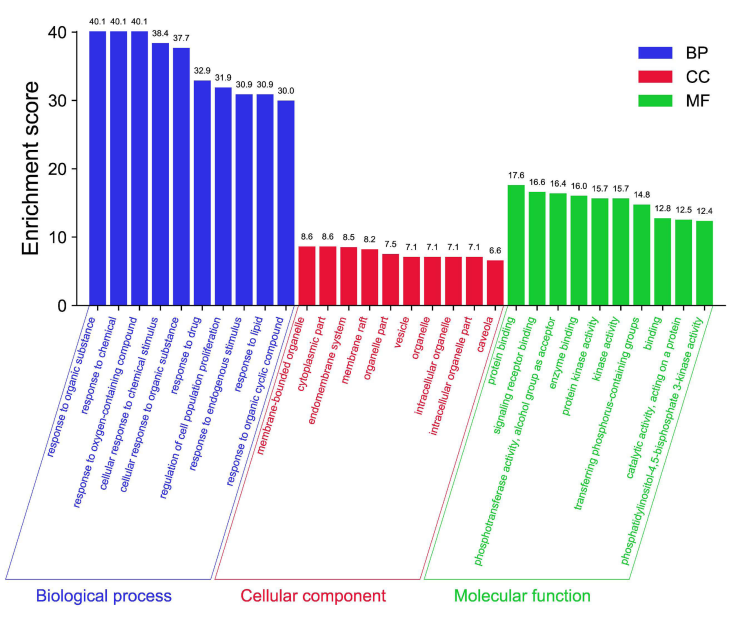

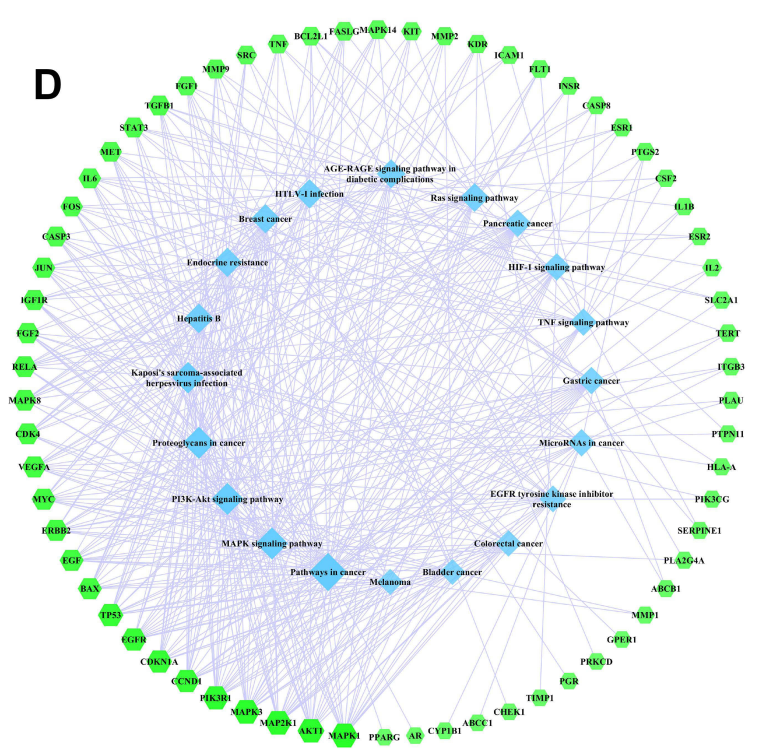

Figure 5 PPI network analysis and enrichment analysis. (A) The PPI network. The size and color changes of the nodes are determined by the degree value. The degree value presented in a descending order (large to small) corresponds to the size in a descending order (large to small) and the color spectrum ranging from bright to dark intensity. The changes of the edge width and color are determined by the combined score, which follow the same principle as the nodes. (B) GO enrichment. The X-axis represents the description of the items and the Y-axis represents the enrichment score (the negative logarithm of the p-value). (C) KEGG pathway enrichment. The size of the bubble in the graph indicates the number of common targets enriched in the pathway and the p-value in a descending order (large to small) corresponds to the bubble color spectrum ranging from blue to red. The $\mathrm{X}$-axis represents the gene ratio and the $\mathrm{Y}$-axis represents the description of the pathways. (D) The target-pathway network. The greencolored nodes in the figure represent the targets and the blue-colored nodes represent the pathways.

\section{Western Blotting}

The expression of p-ERK (dilution ratio 1:2000), ERK (1:2000), p-JNK (1:1000), JNK (1:1500), p-p38 (1:1000), and p38 (1:1000) were determined by Western blotting to investigate the changes in the MAPK signaling pathway. Meanwhile, the levels of p-AKT (1:2000) and AKT (1:3000) were determined to verify the PI3K-AKT signaling pathway. GAPDH (1:2000) was used as an internal control, and the results have been illustrated in Figure 7A.
The results of the statistical analysis showed that there was no statistically significant difference in the expression of ERK, JNK, p38, and AKT between the groups (Figure 7B; $\mathrm{P}>0.05$ ), indicating that the administration of QLP exerted no significant effect on the total amount of proteins. After treatment, the levels of p-ERK/ERK, p-JNK/JNK, p-p38/p38, and p-AKT/AKT declined. Compared with the model control group, the expression levels of p-ERK/ERK, p-p38/p38, and p-AKT/AKT in the 

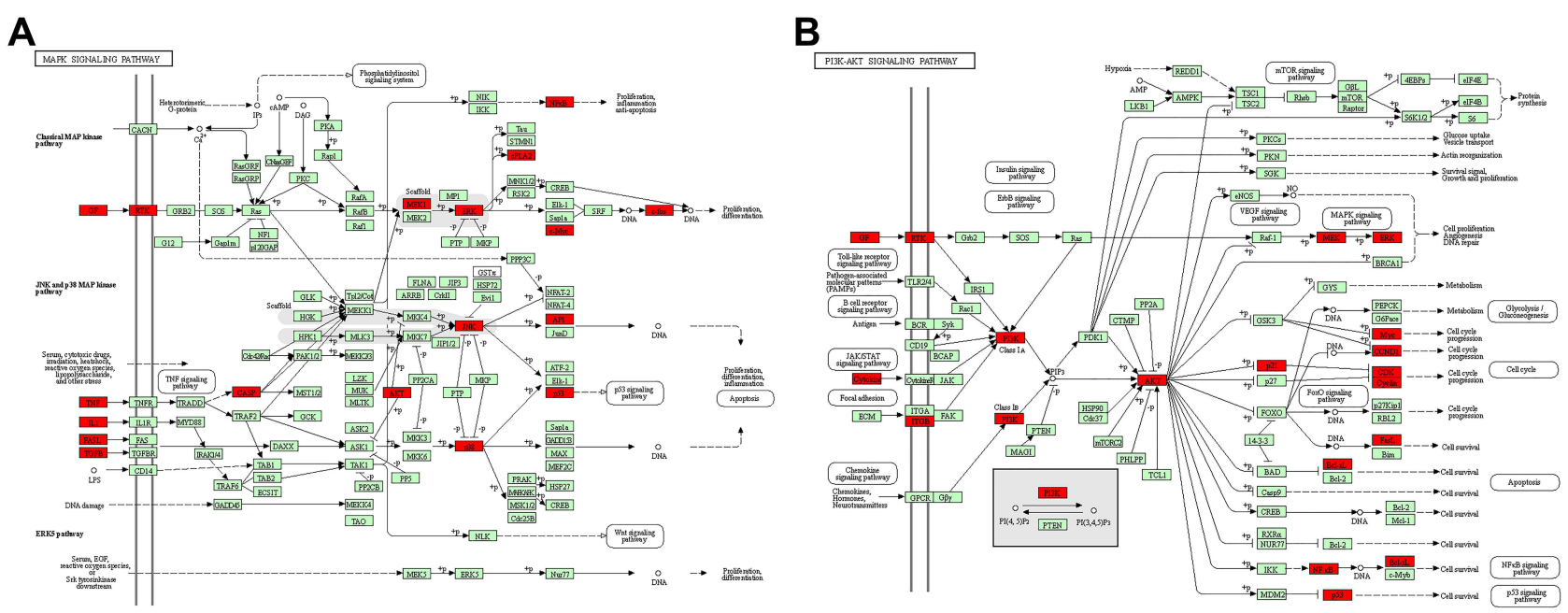

Figure 6 Common targets (highlighted in red) that enriched in the MAPK and PI3K-AKT signaling pathways. (A) MAPK signaling pathway in KEGG database. (B) PI3K-AKT signaling pathway in KEGG database.

QLP-M and QLP-H groups demonstrated the achievement of statistical significance (Figure 7B; $\mathrm{P}<0.01, \mathrm{P}<0.05$ ). These results indicated that QLP reduced the phosphorylation level of proteins and inhibited the excessive activation of the MAPK and PI3K-AKT signaling pathways.

\section{Molecular Docking}

For the MAPK and PI3K-AKT pathways, a compoundtarget-pathway network was constructed using Cytoscape 3.7.2 consisting of 77 nodes and 243 edges (Figure 8). Based on the network, it can be inferred that 37 QLP components and 38 common targets are involved in the two pathways.

Based on the degree value, betweenness centrality, and closeness centrality of the network nodes, malonyl ursolic acid hermiester, emodin, icaritin, tangeretin, and sinensetin were selected as key compounds, and KDR, EGFR, PIK3CG, AKT1, and VEGFA were identified as key targets (Table 1). According to the findings reported by previous research, sorafenib, ${ }^{29}$ erlotinib, ${ }^{30}$ duvelisib, ${ }^{31}$ resveratrol, ${ }^{32}$ and minocycline ${ }^{33}$ were used as positive controls for the ligands of KDR, EGFR, PIK3CG, AKT1, and VEGFA, respectively.

Based on the relationship between the components and the corresponding predicted targets, 15 combinations of key compounds and targets were obtained. Molecular docking results showed that all combinations presented with appreciable binding results (Table 2). Therefore, the selected compounds were found to bind better to the targets enriched in the MAPK and PI3K-AKT signaling pathways, thereby enhancing their regulatory role.

\section{Discussion}

Many causes of POI development have been reported. In this study, we established a rat model of POI by conducting bilateral partial ovariectomy. Ovarian surgery, such as oophorectomy and oophorocystectomy, may lead to the occurrence of POI. ${ }^{34-38}$ It has been shown that ovariectomy exerts less effect on the general condition of animals, while POI symptoms are significant, which has been conducive to subsequent studies. ${ }^{39}$ Therefore, we used bilateral partial ovariectomy to remove $3 / 4$ th portion of the bilateral ovaries from experimental rats to construct a POI model. Post-operation, the estrous cycle of the rats was found to be disordered, exhibiting a decreased number of complete cycles, indicating the successful establishment of the POI model.

We explored the pharmacological effects of QLP on ovarian function from the perspectives of the estrous cycle, hormone levels, and follicle count. The results showed that, after completion of 4 weeks of administration of QLP, the number of estrus cycles in the QLP-M and QLP-H groups increased, indicating the recovery of the rat estrus cycle. Regarding hormone levels, no statistical difference was observed in $\mathrm{E}_{2}$ levels between the different groups; however, the FSH levels of the QLP-M and QLP-H groups were found to be significantly reduced. Additionally, the number of primordial follicles and total follicles was significantly decreased in the model group, which might be due to the accelerated depletion of follicles caused by ovarian surgery. In contrast, administration with QLP significantly decelerated follicle depletion to enhance the ovarian reserve. Kawamura reported that the fragmentation of ovaries disrupted the Hippo signaling 
A

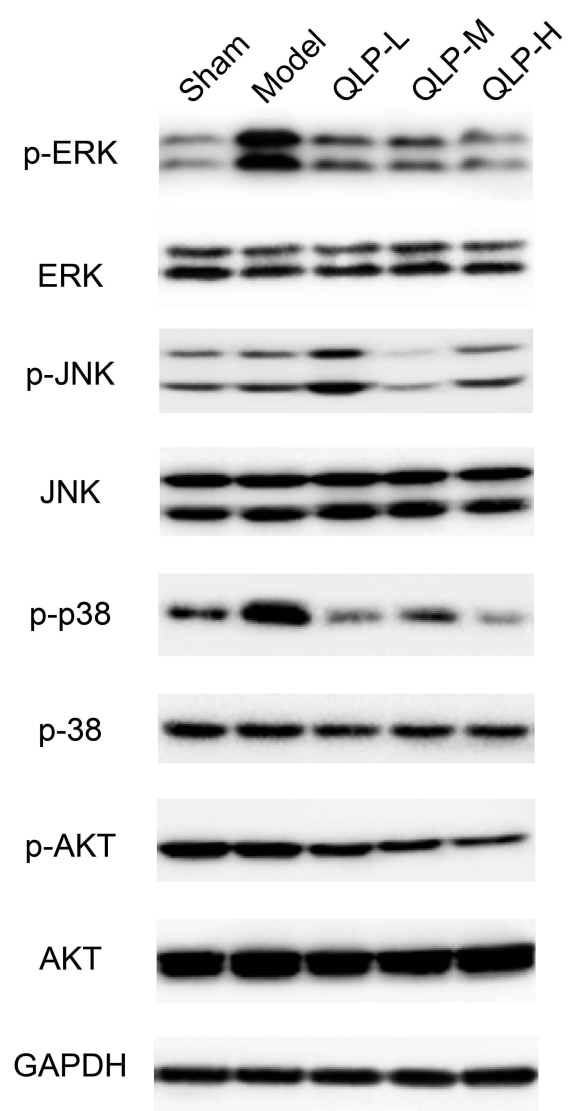

B
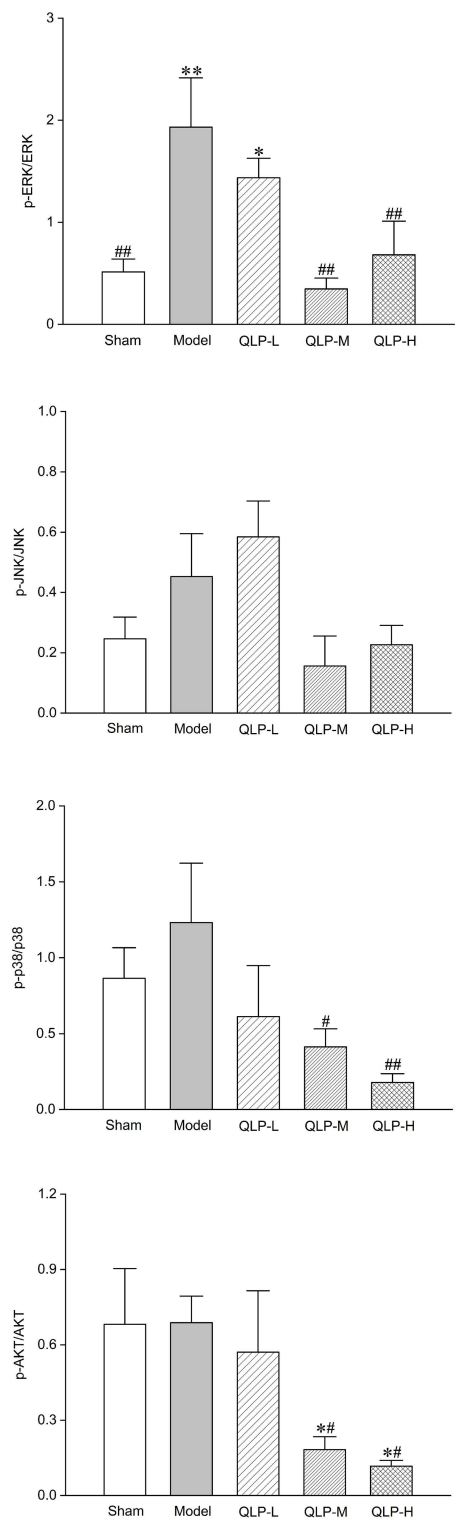
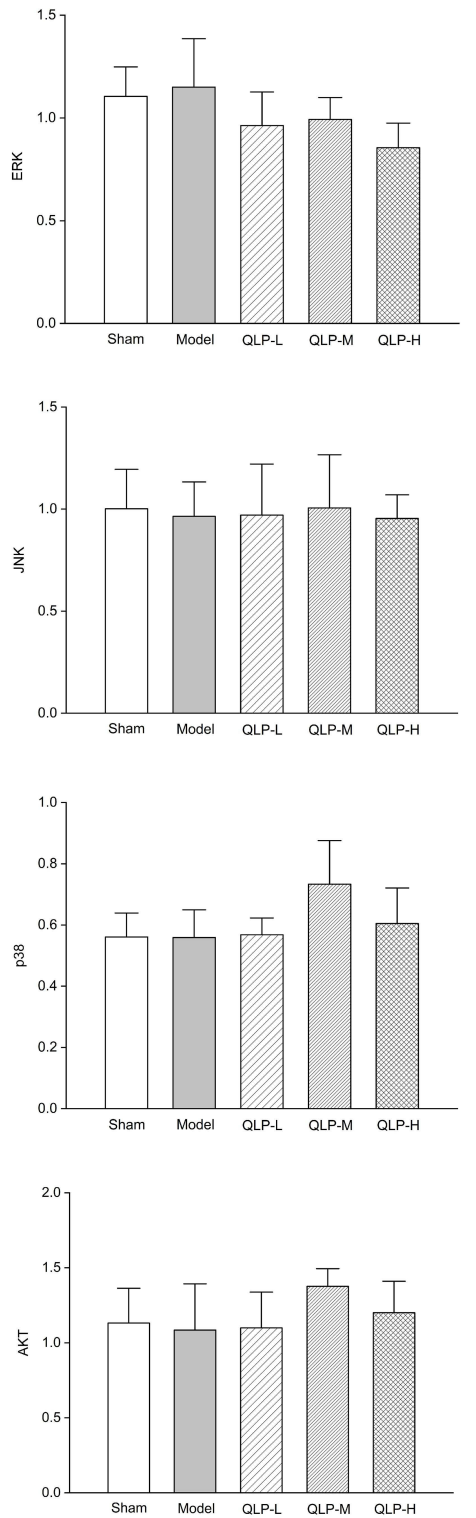

Figure 7 Western blot analysis. (A) Expression levels of p-ERK, ERK, p-JNK, JNK, p-p38, p38, p-AKT, and AKT in the rat ovaries deduced by Western blot analysis. (B)

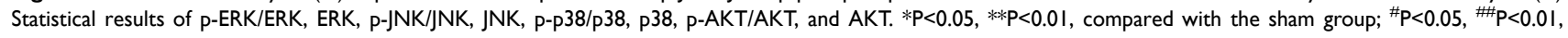
compared with the model group; four animals in each group were determined.

pathway, leading to the promotion of follicle growth and the generation of mature oocytes. ${ }^{10,40}$ Therefore, the balance in follicular development may be disrupted after bilateral partial ovariectomy, resulting in excessive follicular activation and accelerated depletion.

In the present study, three dosage groups of QLP were evaluated, namely low, medium, and high. The results showed that all dosage groups exhibited improvement in ovarian function, and the QLP-M group demonstrated the best therapeutic effect in the treatment of POI rats.

Since QLP is a Chinese medicine compound composed of 15 herbs, if all the compounds contained in each herb were included, the network would not have emphasized the key points in the subsequent study conducted on the therapeutic mechanism. Furthermore, consideration of all compounds would increase the probability of deviation from the actual effects. Therefore, we directly considered the conclusions of the literature reported by Tang et al. ${ }^{18}$ They orally administrated QLP to rats to comprehensive investigate the chemical constituents of QLP in vivo using a method based on UPLC/Q-TOF-MS. As a result, a total of 76 prototype components of QLP in rat bio-samples, including 22 in plasma, 47 in urine, 27 in bile and 51 in feces were identified or tentatively characterized. 


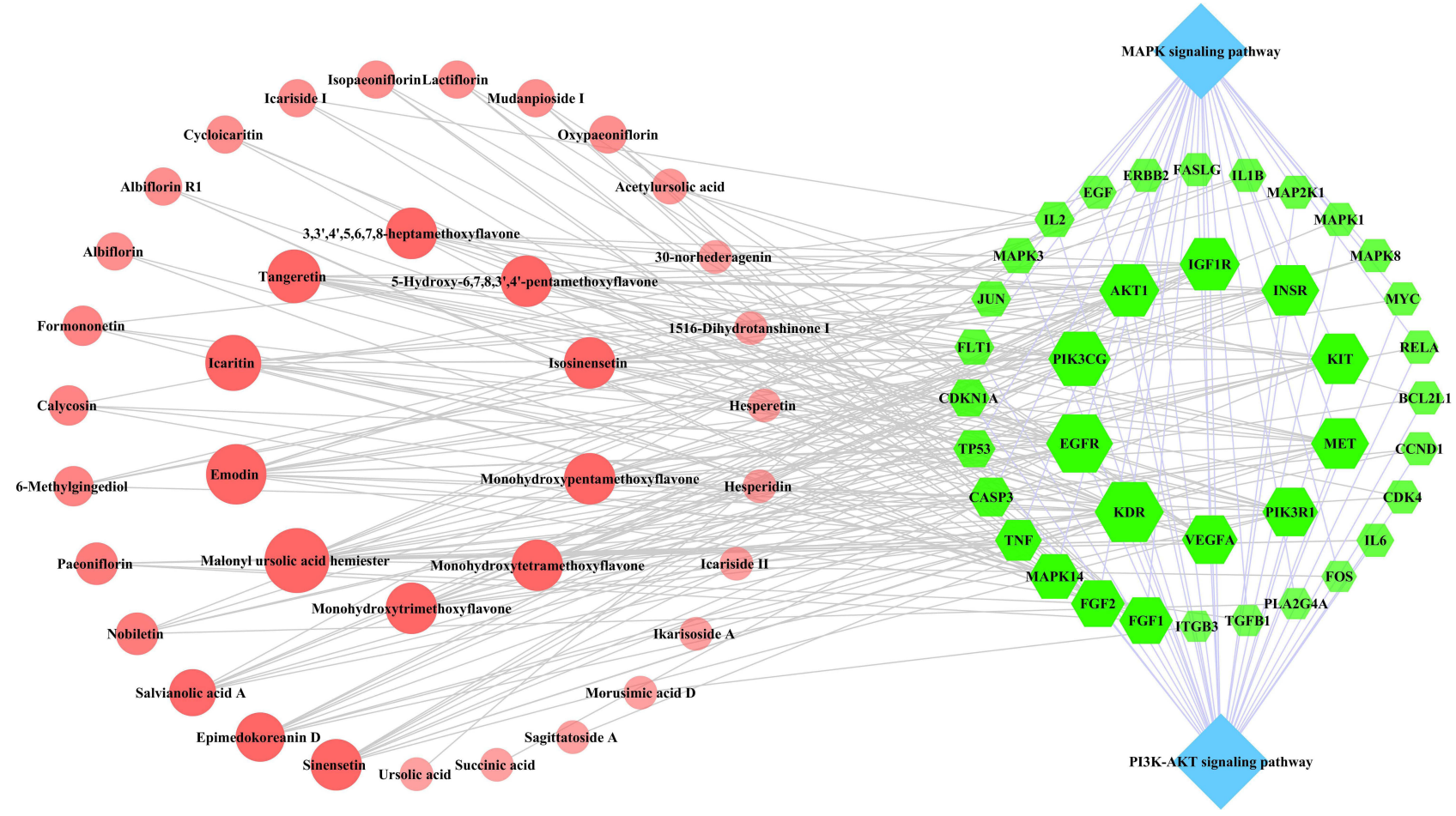

Figure 8 Compound-target-MAPK and the PI3K-AKT pathway network. The green-colored nodes in the network represent the common targets, the blue-colored nodes represent the MAPK and PI3K-AKT pathways, and the light red-colored nodes represent the compounds of QLP. The size of the nodes in the network are determined by the degree value.

Additionally, the compounds of QLP in this study were identified from rat bio-samples, so the prediction of the pharmacokinetics of each compound was unnecessary.

We combined the SwissTargetPrediction database and TCMSP when screening QLP targets. Swisstargetprediction database predicts the target of compounds based on the similarity of two or three-dimensional chemical structures of known compounds, and provides a correlation score for each predicted target. ${ }^{41}$ The TCMSP is a classical database for systematic study of traditional Chinese medicine components and targets. ${ }^{42}$
The DrugBank database, DisGeNET database, and GeneCards database contain a large amount of disease target information that has been reported or has been clinically applied. After obtaining the data on the drug and disease targets, we constructed a compound-common target network. Based on the analysis of the network, 49 components of QLP were found to be related to the treatment of POI, of which eight were shared by more than one herb. Majority of the components were flavonoids, phenolic acids, triterpenoids, and monoterpenes, with flavonoids being the predominant compounds.

Table I The Attribute Value of Key Compounds and Key Targets

\begin{tabular}{|l|c|c|c|c|}
\hline & Name & Degree & Betweenness Centrality & Closeness Centrality \\
\hline Compound & Malonyl ursolic acid hemiester & 15 & 0.06078 & 0.3850 \\
& Emodin & 13 & 0.05087 & 0.4053 \\
& Icaritin & 11 & 0.01059 & 0.3738 \\
& Tangeretin & 10 & 0.01251 & 0.3775 \\
& Sinensetin & 9 & 0.004627 & 0.3702 \\
\hline \multirow{2}{*}{ Target } & KDR & 17 & 0.09683 & 0.4812 \\
& EGFR & 16 & 0.07036 & 0.4753 \\
& PIK3CG & 14 & 0.06804 & 0.4425 \\
& AKTI & 13 & 0.03286 & 0.4583 \\
& VEGFA & 11 & 0.07319 & 0.4477 \\
\hline
\end{tabular}


Table 2 Docking Results of the Key Compounds with the Key Targets

\begin{tabular}{|l|c|c|c|c|c|}
\hline Target & PDB ID & Compound & Affinity (kcal/mol) & Positive Control & Affinity of Positive Control (kcal/mol) \\
\hline KDR & 2XIR & Emodin & -7.4 & Sorafenib & -8.0 \\
KDR & 2XIR & Icaritin & -7.8 & Sorafenib & -8.0 \\
KDR & 2XIR & Sinensetin & -7.3 & Sorafenib & -8.0 \\
KDR & 2XIR & Tangeretin & -7.1 & Sorafenib & -8.0 \\
EGFR & 5YU9 & Emodin & -8.5 & Erlotinib & -8.1 \\
EGFR & 5YU9 & Icaritin & -8.3 & Erlotinib & -8.1 \\
EGFR & 5YU9 & Sinensetin & -7.4 & Erlotinib & -8.1 \\
EGFR & 5YU9 & Tangeretin & -7.5 & Erlotinib & -8.1 \\
PIK3CG & 2CHX & Emodin & -8.5 & Duvelisib & -9.4 \\
PIK3CG & 2CHX & Icaritin & -9.1 & Duvelisib & -9.4 \\
PIK3CG & 2CHX & Sinensetin & -8.1 & Duvelisib & -9.4 \\
PIK3CG & 2CHX & Tangeretin & -7.8 & Duvelisib & -9.4 \\
AKTI & IUNQ & Icaritin & -6.2 & Resveratrol & -6.0 \\
AKTI & IUNQ & Sinensetin & -5.9 & Resveratrol & -6.0 \\
VEGFA & 3QTK & Malonyl ursolic acid hemiester & -8.2 & Minocycline & -8.4 \\
\hline
\end{tabular}

Previous studies have shown that flavonoids have potential therapeutic effects on POIs. For example, icariin can attenuate ovarian injury by promoting DNA damage repair to exert a protective effect against d-galactoseinduced premature ovarian failure, ${ }^{43}$ and chrysin ameliorates radiation-induced POI by downregulating the TGF- $\beta$ / MAPK signaling pathways. ${ }^{44}$ These results were consistent with our findings, which showed that flavonoids in QLP played an important role in the treatment of POI. Furthermore, it can be implied from the network that the key herbs included citri reticulatae pericarpium viride, paeoniae radix alba, epimedii folium, salviae miltiorrhizae radix et rhizoma, cynomorii herba and mori fructus. These results provide a basic chemical foundation for elucidating the mechanism of QLP in the treatment of POI.

To further investigate the interaction relationships of common targets and the biological processes involved, PPI network and enrichment analysis were performed using the STRING database. As a result, STAT3, SRC, MAPK1, and MAPK3 were found to be critical to the PPI network. The transcriptional regulator STAT3 plays key roles in inflammation and immunity, where either the hyperactivation or inactivation of STAT3 results in the development of human diseases. ${ }^{45}$ SRC is a regulatory protein that plays roles in cell differentiation, motility, proliferation, and survival. $^{46}$ MAPK1/ERK2 and MAPK3/ERK1 are the two MAPKs in the MAPK/ERK cascade that mediate diverse biological functions, including cell growth, adhesion, survival, and differentiation.
Based on the results of the KEGG enrichment analysis, we validated the roles of the MAPK and PI3K-AKT signaling pathways. The MAPK signaling pathway is involved in the regulation of cell proliferation, differentiation, and apoptosis, and the activation of MAPKs has recently been found to be associated with POI development. ${ }^{44,47}$ The PI3K-AKT signaling pathway has a wide range of functions, and plays an important role in the regulation of follicle activation and the development of POI. ${ }^{48-51}$ The hyperactivation of the PI3KAKT signaling pathway will rapidly deplete the number of primordial follicles and result in POI. ${ }^{48,52}$ The results showed that there were no significant differences in the expression of ERK, JNK, p38, and AKT between the groups. However, the levels of p-ERK/ERK, p-JNK/ JNK, p-p38/p38, and p-AKT/AKT were decreased, indicating that QLP inhibited the excessive activation of MAPK and PI3K-AKT signaling pathways to alleviate the depletion of follicles. Notably, a key consensus in the understanding of the depletion of the ovarian reserve is that primordial follicles cannot be regenerated or replaced $;^{53}$ therefore, a reasonable explanation is that QLP decelerated the process of follicular depletion rather than increasing the number of primordial follicles.

Finally, to further confirm the effect of QLP on the MAPK and PI3K-AKT signaling pathways from another perspective, we validated the key compounds of QLP and the key targets enriched in the two signaling pathways in the compound-target-pathway network by molecular 
docking. We concluded that malonyl ursolic acid hemiester, emodin, icaritin, tangeretin, and sinensetin were the key compounds, while KDR, EGFR, PIK3CG, AKT1, and VEGFA were the key targets. Malonyl ursolic acid hemiester is a triterpenoid derived from cynomorii herba, while emodin is an anthraquinone derived from polygoni multiflori radix preparata. Icaritin, tangeretin, and sinensetin are flavonoids. Icaritin is derived from epimedii folium, while tangeretin and sinensetin are derived from citri reticulatae pericarpium viride. In this study, positive controls were used to assess the binding ability, while some previous studies have used an empirical threshold of -5 $\mathrm{kcal} / \mathrm{mol}^{54-56}$ Regardless of the evaluation standard used, it was concluded that the key compounds of QLP demonstrated good binding ability with the key targets, further illustrating the existence of regulation of the MAPK and PI3K-AKT signaling pathways.

\section{Conclusion}

In this study, we investigated the therapeutic effects of QLP exerted on resection-induced POI in rats and systematically explored the key mechanisms involved. The results showed that QLP could significantly improve ovarian function by promoting the recovery of the estrus cycle, by regulating the FSH levels, and by enhancing ovarian reserves. The optimal dose of QLP in the experiment was found to be $0.9 \mathrm{~g} / \mathrm{kg}$.

Based on the network pharmacology method prescribed for traditional Chinese medicine compounds combined with validation, we investigated the pharmacological mechanism of QLP in the treatment of POI to elucidate the chemical constituents and biological processes involved. Experimental verification and molecular docking showed that QLP inhibited the excessive activation of the MAPK and PI3K-AKT signaling pathways to exert its therapeutic effects. These findings provide novel insights into QLP for application in the clinical treatment of POI.

\section{Acknowledgments}

This study was supported by funding from the National Major Scientific and Technological Special Project for "Significant New Drugs Development" during the Thirteenth Five-Year Plan Period of China (Grant number 2018ZX09201017-002).

\section{Disclosure}

The authors report no conflicts of interest in this work.

\section{References}

1. Rossetti R, Ferrari I, Bonomi M, Persani L. Genetics of primary ovarian insufficiency. Clin Genet. 2017;91(2):183-198. doi:10.1111/ cge. 12921

2. Webber L, Davies M; European Society for Human Reproduction and Embryology (ESHRE) Guideline Group on POI. ESHRE Guideline: management of women with premature ovarian insufficiency. Hum Reprod. 2016;31(5):926-937. doi:10.1093/humrep/dew027.

3. De Vos M, Devroey P, Fauser BC. Primary ovarian insufficiency. Lancet. 2010;376(9744):911-921. doi:10.1016/s0140-6736(10)60355-8

4. Vujovic S. Aetiology of premature ovarian failure. Menopause Int. 2009;15(2):72-75. doi:10.1258/mi.2009.009020

5. Welt CK. Primary ovarian insufficiency: a more accurate term for premature ovarian failure. Clin Endocrinol. 2008;68(4):499-509. doi:10.1111/j.1365-2265.2007.03073.x

6. Ahmadian S, Mahdipour M, Pazhang M, et al. Effectiveness of stem cell therapy in the treatment of ovarian disorders and female infertility: a systematic review. Curr Stem Cell Res Ther. 2020;15 (2):173-186. doi:10.2174/1574888X14666191119122159

7. Ulin M, Cetin E, Hobeika E, et al. Human mesenchymal stem cell therapy and other novel treatment approaches for premature ovarian insufficiency. Reprod Sci. 2021;28(6):1688-1696. doi:10.1007/ s43032-021-00528-z

8. Hajipour H, Farzadi L, Latifi Z, et al. An update on platelet-rich plasma (PRP) therapy in endometrium and ovary related infertilities: clinical and molecular aspects. Syst Biol Reprod Med. 2021;67 (3):177-188. doi:10.1080/19396368.2020.1862357

9. Ahmadian S, Sheshpari S, Pazhang M, et al. Intra-ovarian injection of platelet-rich plasma into ovarian tissue promoted rejuvenation in the rat model of premature ovarian insufficiency and restored ovulation rate via angiogenesis modulation. Reprod Biol Endocrinol. 2020;18 (1):78. doi:10.1186/s12958-020-00638-4

10. Kawamura K, Cheng Y, Suzuki N, et al. Hippo signaling disruption and Akt stimulation of ovarian follicles for infertility treatment. Proc Natl Acad Sci U S A. 2013;110(43):11479-17474. doi:10.1073/ pnas. 1312830110

11. Zhai J, Yao G, Dong F, et al. In vitro activation of follicles and fresh tissue auto-transplantation in primary ovarian insufficiency patients. J Clin Endocrinol Metab. 2016;101(11):4405-4412. doi:10.1210/ jc.2016-1589

12. Zhao H, Shan Y, Ma Z, Yu M, Gong B. A network pharmacology approach to explore active compounds and pharmacological mechanisms of epimedium for treatment of premature ovarian insufficiency. Drug Des Devel Ther. 2019;13:2997-3007. doi:10.2147/DDDT.S207823

13. Li Y, Xia G, Tan Y, Shuai J. Acupoint stimulation and Chinese herbal medicines for the treatment of premature ovarian insufficiency: a systematic review and meta-analysis. Complement Ther Clin Pract. 2020;41:101244. doi:10.1016/j.ctcp.2020.101244

14. Liu HD, Yin YH. Qilin pills prevent ovarian hyperstimulation syndrome of patients after IVF-ET by regulating plasma-related indicators [Article in Chinese]. Chin J Clin Pharmacol Ther. 2020;25 (02):196-202. doi:10.12092/j.issn.1009-2501.2020.02.012

15. Liao HY, Gao J, Qiu P, et al. Qilin pills in the treatment of 60 cases of ovarian reserve decline-induced irregular menstruation: a multicenter, single-blinded, randomize-controlled clinical trial [Article in Chinese]. J Tradit Chin Med. 2019;60(24):2107-2112. doi:10.13288/j.11-2166/r.2019.24.010

16. Liao HY, Gao J, Qiu P, et al. Clinical observation of qilin pills in treating irregular menstruation [Article in Chinese]. J Guangzhou Univ Tradit Chin Med. 2019;36(12):1911-1915. doi:10.13359/j. cnki.gzxbtcm.2019.12.009

17. Li S, Zhang ZQ, Wu LJ, Zhang XG, Li YD, Wang YY. Understanding ZHENG in traditional Chinese medicine in the context of neuro-endocrine-immune network. IET Syst Biol. 2007;1 (1):51-60. doi:10.1049/iet-syb:20060032 
18. Tang XY, Zeng JX, Dai ZQ, et al. Identification and characterization of chemical constituents in Qi-Lin pills and their metabolites in rat bio-samples after oral administration using ultra-high performance liquid chromatography with quadrupole time-of-flight mass spectrometry. J Pharm Biomed Anal. 2020;188:113402. doi:10.1016/j.jpba.2020.113402

19. National Research Council (US) Committee for the Update of the Guide for the Care and Use of Laboratory Animals. Guide for the Care and Use of Laboratory Animals. 8th ed. Washington (DC): National Academies Press (US); 2011.

20. Wei W, Wu XM, Li YJ. Experimental Methodology of Pharmacology. 4th ed. Beijing: People's Medical Publishing House; 2010.

21. Yang Z, Du X, Wang C, et al. Therapeutic effects of human umbilical cord mesenchymal stem cell-derived microvesicles on premature ovarian insufficiency in mice. Stem Cell Res Ther. 2019;10(1):250. doi:10.1186/s13287-019-1327-5

22. Feng X, Ling L, Zhang W, et al. Effects of human amnion-derived mesenchymal stem cell (hAD-MSC) transplantation in situ on primary ovarian insufficiency in SD rats. Reprod Sci. 2020;27 (7):1502-1512. doi:10.1007/s43032-020-00147-0

23. Cora MC, Kooistra L, Travlos G. Vaginal cytology of the laboratory rat and mouse: review and criteria for the staging of the estrous cycle using stained vaginal smears. Toxicol Pathol. 2015;43(6):776-793. doi: $10.1177 / 0192623315570339$

24. Fu X, He Y, Wang X, et al. Overexpression of miR-21 in stem cells improves ovarian structure and function in rats with chemotherapy-induced ovarian damage by targeting PDCD4 and PTEN to inhibit granulosa cell apoptosis. Stem Cell Res Ther. 2017;8(1):187. doi:10.1186/s13287-017-0641-z

25. Kaplan S, Türk A. Adalimumab increases follicle reserve and follicle development in rat ovary: the effect of adalimumab on ovarian reserve. Cureus. 2020;12(10):e11230. doi:10.7759/cureus.11230

26. Sanner MF. Python: a programming language for software integration and development. J Mol Graph Model. 1999;17(1):57-61.

27. Trott O, Olson AJ. Autodock Vina: improving the speed and accuracy of docking with a new scoring function, efficient optimization, and multithreading. J Comput Chem. 2010;31:455-461. doi:10.1002/ jcc. 21334

28. Li DB, Zhou L, Sun ZY. Research advances of premature ovarian insufficiency-related signaling pathways [Article in Chinese]. Chin $J \quad$ Comp Med. 2020;30(2):121-127. doi:10.3969/j.issn.16717856.2020.02.019

29. El-Helby AA, Sakr H, Eissa IH, Abulkhair H, Al-Karmalawy AA, El-Adl K. Design, synthesis, molecular docking, and anticancer activity of benzoxazole derivatives as VEGFR-2 inhibitors. Arch Pharm. 2019;352(10):e1900113. doi:10.1002/ardp.201900113

30. Park JH, Liu Y, Lemmon MA, Radhakrishnan R. Erlotinib binds both inactive and active conformations of the EGFR tyrosine kinase domain. Biochem J. 2012;448(3):417-423. doi:10.1042/BJ20121513

31. Vangapandu HV, Jain N, Gandhi V. Duvelisib: a phosphoinositide-3 kinase $\delta / \gamma$ inhibitor for chronic lymphocytic leukemia. Expert Opin Investig Drugs. 2017;26(5):625-632. doi:10.1080/ 13543784.2017.1312338

32. Hsieh TC, Lin CY, Bennett DJ, Wu E, Wu JM. Biochemical and cellular evidence demonstrating AKT-1 as a binding partner for resveratrol targeting protein NQO2. PLoS One. 2014;9(6):e101070. doi:10.1371/journal.pone.0101070

33. Yao JS, Chen Y, Zhai W, Xu K, Young WL, Yang GY. Minocycline exerts multiple inhibitory effects on vascular endothelial growth factor-induced smooth muscle cell migration: the role of ERK1/2, PI3K, and matrix metalloproteinases. Circ Res. 2004;95(4):364-371. doi:10.1161/01.RES.0000138581.04174.2f

34. Słopień R. Mood disorders in women with premature ovarian insufficiency. Prz Menopauzalny. 2018;17(3):124-126. doi:10.5114/ pm.2018.78556
35. Ishizuka B. Current Understanding of the Etiology, Symptomatology, and Treatment Options in Premature Ovarian Insufficiency (POI). Front Endocrinol. 2021;12:626924. doi:10.3389/fendo.2021.626924

36. Jin M, Yu Y, Huang H. An update on primary ovarian insufficiency. Sci China Life Sci. 2012;55(8):677-686. doi:10.1007/s11427-012-4355-2

37. Rosendahl M, Simonsen MK, Kjer JJ. The influence of unilateral oophorectomy on the age of menopause. Climacteric. 2017;20 (6):540-544. doi:10.1080/13697137.2017.1369512

38. Cheng JJ, Ruan XY, Zhou Q, et al. Study on the effect of the ovarian tissue volume on estrous cycle and body weight in rats [Article in Chinese]. J Capit Univ Med Sci. 2019;40(04):554-559. doi:10.3969/j. issn.1006-7795.2019.04.012

39. Zhou Y, Jia YL, Yan DW, et al. Comparison of two kinds of premature ovarian failure model in mice [Article in Chinese]. Shanghai Med J. 2018;41(08):489-494.

40. Hsueh AJW, Kawamura K. Hippo signaling disruption and ovarian follicle activation in infertile patients. Fertil Steril. 2020;114 (3):458-464. doi:10.1016/j.fertnstert.2020.07.031

41. Daina A, Michielin O, Zoete V. SwissTargetPrediction: updated data and new features for efficient prediction of protein targets of small molecules. Nucleic Acids Res. 2019;47(W1):W357-W364. doi:10.1093/nar/gkz382

42. Ru J, Li P, Wang J, et al. TCMSP: a database of systems pharmacology for drug discovery from herbal medicines. J Cheminform. 2014;6:13. doi:10.1186/1758-2946-6-13

43. Li N, Wang J, Wang X, Sun J, Li Z. Icariin exerts a protective effect against d-galactose induced premature ovarian failure via promoting DNA damage repair. Biomed Pharmacother. 2019;118:109218. doi:10.1016/j.biopha.2019.109218

44. Mantawy EM, Said RS, Abdel-Aziz AK. Mechanistic approach of the inhibitory effect of chrysin on inflammatory and apoptotic events implicated in radiation-induced premature ovarian failure: emphasis on TGF- $\beta$ /MAPKs signaling pathway. Biomed Pharmacother. 2019;109:293-303. doi:10.1016/j.biopha.2018.10.092

45. Hillmer EJ, Zhang H, Li HS, Watowich SS. STAT3 signaling in immunity. Cytokine Growth Factor Rev. 2016;31:1-15. doi:10.1016/ j.cytogfr.2016.05.001

46. Roskoski R Jr. Src kinase regulation by phosphorylation and dephosphorylation. Biochem Biophys Res Commun. 2005;331 (1):1-14. doi:10.1016/j.bbrc.2005.03.012

47. Liu T, Lin J, Chen C, et al. MicroRNA-146b-5p overexpression attenuates premature ovarian failure in mice by inhibiting the Dab2ip/Ask1/p38-Mapk pathway and $\gamma \mathrm{H} 2 \mathrm{~A} . \mathrm{X}$ phosphorylation. Cell Prolif. 2021;54(1):e12954. doi:10.1111/cpr.12954

48. John GB, Gallardo TD, Shirley LJ, Castrillon DH. Foxo3 is a PI3K-dependent molecular switch controlling the initiation of oocyte growth. Dev Biol. 2008;321(1):197-204. doi:10.1016/j.ydbio. 2008.06.017

49. Balakrishnan B, Nicholas C, Siddiqi A, et al. Reversal of aberrant PI3K/Akt signaling by Salubrinal in a GalT-deficient mouse model. Biochim Biophys Acta Mol Basis Dis. 2017;1863(12):3286-3293. doi:10.1016/j.bbadis.2017.08.023

50. Zhang H, Qin F, Liu A, et al. Electro-acupuncture attenuates the mice premature ovarian failure via mediating PI3K/AKT/mTOR pathway. Life Sci. 2019;217:169-175. doi:10.1016/j.lfs.2018.11.059

51. Li N, Liu L. Mechanism of resveratrol in improving ovarian function in a rat model of premature ovarian insufficiency. J Obstet Gynaecol Res. 2018;44(8):1431-1438. doi:10.1111/jog. 13680

52. Chang EM, Lim E, Yoon S, et al. Cisplatin induces overactivation of the dormant primordial follicle through PTEN/AKT/FOXO3a pathway which leads to loss of ovarian reserve in mice. PLoS One. 2015;10(12):e0144245. doi:10.1371/journal.pone.0144245

53. Anderson RA, Wallace WHB, Telfer EE. Ovarian tissue cryopreservation for fertility preservation: clinical and research perspectives. Hum Reprod Open. 2017;2017(1):hox001. doi:10.1093/hropen/ hox001 
54. Zhang J, Liu X, Zhou W, et al. A bioinformatics investigation into molecular mechanism of Yinzhihuang granules for treating hepatitis B by network pharmacology and molecular docking verification. Sci Rep. 2020;10(1):11448. doi:10.1038/s41598-020-68224-7

55. Huang ZZ, Du X, Ma CD, Zhang RR, Gong WL, Liu F. Identification of antitumor active constituents in polygonatum sibiricum flower by UPLC-Q-TOF-MSE and network pharmacology. ACS Omega. 2020;5(46):29755-29764. doi:10.1021/acsomega.0c03582
56. Liu F, Li Y, Yang Y, et al. Study on mechanism of matrine in treatment of COVID-19 combined with liver injury by network pharmacology and molecular docking technology. Drug Deliv. 2021;28(1):325-342. doi:10.1080/10717544.2021.1879313

\section{Publish your work in this journal}

Drug Design, Development and Therapy is an international, peerreviewed open-access journal that spans the spectrum of drug design and development through to clinical applications. Clinical outcomes, patient safety, and programs for the development and effective, safe, and sustained use of medicines are a feature of the journal, which has also been accepted for indexing on PubMed Central. The manuscript management system is completely online and includes a very quick and fair peer-review system, which is all easy to use. Visit http://www. dovepress.com/testimonials.php to read real quotes from published authors.

Submit your manuscript here: https://www.dovepress.com/drug-design-development-and-therapy-journal 